\title{
Searches for Heavy Vector Bosons at CDF
}

\author{
Michael Gold ${ }^{\dagger}$ \\ for the CDF Collaboration \\ ${ }^{\dagger}$ New Mexco Center for Particle Physics \\ University of New Mexico
}

\begin{abstract}
The existence of additional vector bosons are theoretically well motivated, arising naturally in many extensions of the Standard Model. Experimentally, such particles typically have clean signatures and therefore should be observed in hadron colliders if they are produced. We review recent searches for heavy vector bosons with $\mathrm{CDF}$ at the Tevatron and provide limits on their production.
\end{abstract}

Keywords: $Z^{\prime}, W^{\prime}$, gauge boson, CDF, Tevatron

PACS: $12.60 . \mathrm{Cn}$

One of the most compelling arguments for physics beyond the SM is that there is some high-energy unification of the forces of nature. Additional vector bosons $\left(Z^{\prime}, W^{\prime}\right)$ arise naturally in extensions of the standard model (SM) with gauge unification, such as $E_{6}$ and $\mathrm{SO}(10)$ models, as well as in other extensions of the $\mathrm{SM}$, for example models with extra dimensions or little Higgs models. (See reference [1] for a theoretical review of $Z^{\prime}$ models.) If there is left-right symmetry at some high energy scale, then this provides a natural mechanism for light neutrino masses and predicts a (right-handed) $W^{\prime}$. (See reference [2] for a theoretical review of $W^{\prime}$ models.) Experimentally, the production of heavy vector bosons may result in high-mass lepton pair or lepton-neutrino events which provide striking signatures. They may also appear in channels with higher SM backgrounds such as di-jet events, di-boson events and single top events.

Lepton pair production: $\mathrm{CDF}$ has searched for high-mass di-lepton pairs $(e e, \mu \mu)$ in large luminosity samples $\left(2.5 \mathrm{fb}^{-1}\right.$ and $2.3 \mathrm{fb}^{-1}$ respectively). [3, 4] While the background is dominanted by Drell-Yan, in these large data sets backgrounds from QCD (di-jets, $\mathrm{W}+\mathrm{jets}$ ) and other SM process (di-tau, top, di-boson) contribute significantly to the high-mass tail. In the di-electron channel there is a 2.5 sigma fluctuation around 240 $\mathrm{GeV}$ with width consistent with the experimental resolution. Otherwise, the agreement between the data and the SM expectation is spectacular.

The di-electron excess could be evidence for new physics, for example as an indication of new strong dynamics in the model by Cata et al. [5] This theory predicts a narrow spin-1 boson instead of the SM Higgs. However, this theory also has lepton universality, and there is no evidence for a corresponding signal in the di-muon channel. The data has been used to excluded a portion of the paramter space for this model, the ratio of effective couplings versus new boson mass.

The di-electron mass spectrum can be used to set limits on various $Z$ 's (see Figure 1). The theoretical cross sections are given for SM couplings and couplings to $Z^{\prime}$ s from the string-inspired gauge group $E_{6}$. In general, constraints in particular models are weaker

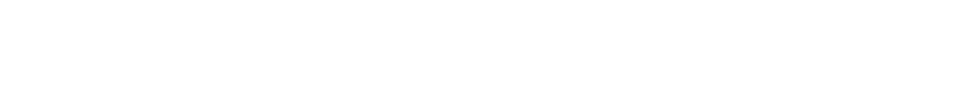


than for SM couplings which is near a TeV.
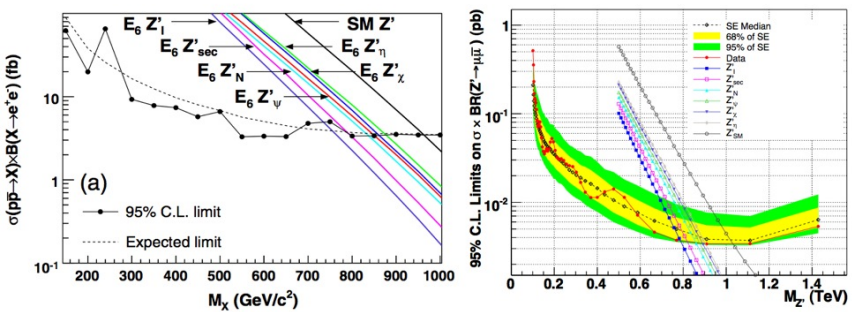

FIGURE 1. Lower limits on $Z^{\prime}$ masses from the measurement of the di-electron (left) and di-muon (right) mass spectrum.

Another kinematic distribution sensitive to a $Z^{\prime}$ is the electron-quark angular distribution in the $Z^{\prime}$ rest frame which gives a forward-backward asymmetry in the laboratory as first pointed out by Rosner.[6] An analysis combining this distribution with the mass distribution allowed CDF to derive a lower limit of $850 \mathrm{GeV}$ (SM couplings) with only $0.45 \mathrm{fb}^{-1}$. [7] One can also use the shape of the high-mass tail to find evidence for a $Z^{\prime}$ where you are not seeing the peak or to search for compositeness (where q,e share constituents). As was recently pointed out by Rizzo this analysis will be less useful at the LHC due to the kinematics of production in proton-proton collisions. [8]

In the di-muon channel it is the distribution of inverse mass which is compared to the SM expectation. The inverse-mass is proportional to the curvature squared and is approximately constant in resolution. A point resolution of 200 microns in the tracking chamber was acheived by precision alignment of the wires to within a few microns using a sample of high- $p_{T}$ cosmic rays for alignment in $r, \phi$ and the E/p distribution from a sample of electrons for alignment in $\mathrm{z}$. The (approximately flat) resolution in inverse mass is $\delta M_{\mu \mu}^{-1} \sim 0.17 \mathrm{TeV}^{-1}$ As for the di-electrons spectrum, small non-DY backgrounds are needed to understand the high-mass tail. Lower limits for various $E_{6}$ $Z^{\prime}$ s as well as a for SM couplings are shown in Figure 1. The SM coupled $Z^{\prime}$ is excluded below $1 \mathrm{TeV}$.

Some further remarks on $Z^{\prime}$ searches with di-leptions. First, a tau-pair search has been done with $0.2 \mathrm{fb}^{-1}$ yielding a lower bound of $M>600 \mathrm{GeV}$ for SM couplings. [10] Second, an interesting class of models that predict a very narrow $Z^{\prime}$ was pointed out by Nath et al. [11] In these models which arise for example in string and super-gravity theories, a $Z^{\prime}$ can appear where the mass arises from a Stueckleberg coupling to a psuedo-scalar. Nath et $a l$. have used CDF and D0 data to constrain a class of these models paramterized by a ratio of mass parameters $(\varepsilon)$ where $\varepsilon=0$ is the SM and small values $\varepsilon \sim 0.05$ are consistent with precision EWK measurements. Using small samples from CDF and D0, they obtain $M>250(375) \mathrm{GeV}$ for $\varepsilon=0.035(0.06)$. This analysis should be redone with high-luminosity data which would have a sensitivity to $\sim 600$ $\mathrm{GeV}$.

Di-jet channels: The highest mass events at the tevatron are the di-jets, which of course also have the highest background. Jets are reconstructed using the midpoint algorithm with $\mathrm{R}=0.7$, and the di-jet spectrum is constructed from central di-jets events with pair masses $>180 \mathrm{GeV} / \mathrm{c}^{2}$. The mass distribution is corrected for jet resolution 

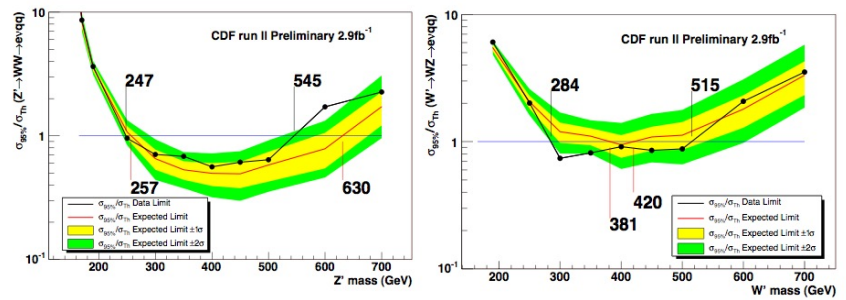

FIGURE 3. Limits on $W^{\prime}, Z^{\prime}$ masses from the di-boson resonance search.

and efficiency. Experimental systematics include the energy scale, mass resolution, and luminosity. The dominant theoretical uncertainty is the uncertainty in the parton distribution functions. Comparing $1.1 \mathrm{fb}^{-1}$ of data to NLO QCD, the agreement is excellent throughout the entire range of the data ( to $\sim 1300 \mathrm{GeV}$ ). In order to perform the resonance search, the data is fit to a smooth curve. The expected $W^{\prime}, Z^{\prime}$ resonance shape is dominated by the jet energy resolution with a low-mass tail from QCD radiation. Limits are extracted for $W^{\prime}, Z^{\prime}$ based on cross section times branching ratio times acceptance (Figure 2).

Di-boson channels: SM di-boson events (WW,WZ,ZZ) can also be produced by decays of a $Z^{\prime}$. CDF has recently published the observation of SM di-bosons in the missing transverse momentum plus di-jets channel.[12] The search does not explicitly require a lepton and is therefore sensitive to both $\ell \bar{v}+j j$ and $v \bar{v}+j j$ final states. A dijet mass resonance from $W \rightarrow j j$ and $Z \rightarrow j j$ decays (not distinguished due to jet energy resolution) is observed with $5.4 \sigma$ significance. In $3.5 \mathrm{fb}^{-1}, 1516 \pm 239 \pm 144$ events are observed in agreement with the SM expectation.

In the $W \rightarrow e \bar{v}+$ jets channel we searched for resonance production of SM boson pairs by reconstructing the $e \bar{v} j j$ mass. Events are selected with di-jet mass consistent with $\mathrm{W}\left(65<M_{j j}<95\right)$ or $\mathrm{Z}\left(75<M_{j j} 105\right)$ decay. The SM background for $\mathrm{WW}(\mathrm{WZ})$ resonance searches are: $\mathrm{Wj} 58.5$ (59.0), QCD 10.7 (10.8) $t \bar{t}$ 9.5 (9.8) WW 7.8 (6.7). In both cases, the data are well reproduced by the SM backgrounds and limits are set on $W^{\prime}, Z^{\prime}$ masses (see Figure 3). The limits are extracted using a summed $(e \bar{v} j j)$ transverse momentum cut (typically $>60 \mathrm{GeV}$ ) that

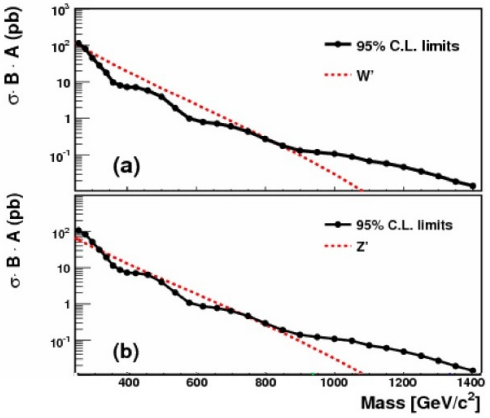

2: Limits from di-jet mass resonance search. is optimized for each $W^{\prime}$ and $Z^{\prime}$ mass search window.

$W^{\prime}$ in single top events: A $W^{\prime}$ would contribute to the single top production channel. Single top has recently been observed by CDF at the $5 \sigma$ level with a $3.2 \mathrm{fb}^{-1}$ sample at a rate consistent with the $\mathrm{SM}$ expectation- $\sigma_{\exp }=2.3_{-0.5}^{+0.6} \mathrm{pb}$ compared to $\sigma_{\text {theo }} \sim$ 
2.9pb. [13] We have also done a direct search for a $W^{\prime}$ in this channel. We search for single top events in the $\mathrm{W}+$ jets channel with one jet having a secondary vertex (b-tagged jet). We observe $1362(1444 \pm 255)$ di-jet events and $617(682 \pm 83)$ three-jet events. The expected $W^{\prime}$ cross section is $\sigma\left(p \bar{p} \rightarrow W^{\prime} \rightarrow t \bar{b}+\bar{t} b\right) \approx 2 \mathrm{pb}$ at $M_{W^{\prime}=600} \mathrm{GeV}$.

Mass limits are set on $W^{\prime}$ with SM coupling based on the cross section times branching ratio limit. $M_{W}^{\prime}>$ $800(825) \mathrm{GeV}$ for $M_{W}^{\prime}>(<) M_{v_{R}}$ where in the later case leptonic decays are not allowed (see Figure 4). This limit improves that obtained from a measurement of the transverse mass line shape in electron plus missing momentum events $(788 \mathrm{GeV}$ with $\left.0.2 \mathrm{fb}^{-1}\right) .[14]$

The table summarizes the CDF limits on $W^{\prime}, Z^{\prime}$ with SM couplings. They are close to the kinematic limit, but 1 remind you that limits in specific models are sig-

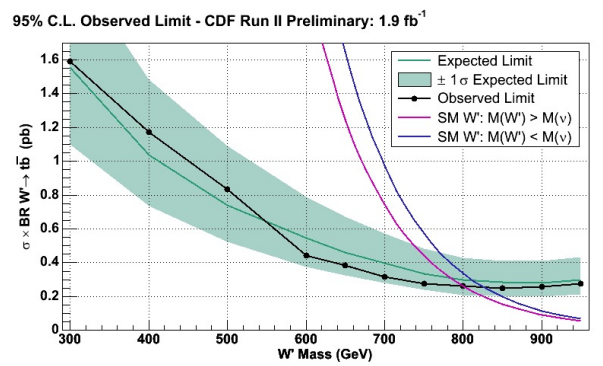
nificantly weaker. We now have $>4 \mathrm{fb}^{-1}$ and there are interesting searches to come.

\begin{tabular}{c|c|cc} 
mode & $\mathrm{L}\left(\mathrm{fb}^{-1}\right)$ & $M_{Z^{\prime}}^{S M}$ & $M_{W^{\prime}}^{S M}$ \\
\hline$e e$ & 2.5 & $150-963$ & \\
$\mu \mu$ & 2.3 & $100-1030$ & \\
$\tau \tau$ & 0.2 & $120-399$ & \\
$\mathrm{jj}$ & 1.1 & $320-740$ & $280-840$ \\
$\mathrm{WW}, \mathrm{WZ}$ & 2.9 & $247-545$ & $284-515$ \\
$t b$ & 1.9 & & $300-950$
\end{tabular}

\section{ACKNOWLEDGMENTS}

M. Gold would like to thank the DOE for its support under award DE-FG02-O4ER41300.

\section{REFERENCES}

1. P. Langacker, Rev. Mod. Phys. 81, 1199Đ1228 (2009) arXiv:0801.1345v2 [hep-ph].

2. Z. Sullivan Phys.Rev.D66:075011,2002

3. Phys. Rev. Lett. 102, 031801 (2009)

4. Phys. Rev. Lett. 102, 091805 (2009)

5. Cata, Isidori, Kamenik arXiv:0905.0490v1

6. J. L. Rosner, Phys. Rev. D54, 1078 (1996).

7. Phys. Rev. Lett. 96211801,2006

8. arXiv:0904.2534

9. Carena, Daleo, Dobrescu, Tait Phys. Rev. D 70-093009 2004

10. Phys. Rev. Lett. 95,131801 (2005)

11. Nath et al. PRL 97:021801,2006

12. arXiv:0905.4714

13. arXiv:0903.0885

14. Phys. Rev. D 75091101 (2007) 